\title{
Computational Explorations of Compatibility and Innovation
}

\author{
Ricardo Sosa' and John S. Gero ${ }^{2}$ \\ 1 Department of Industrial Design, ITESM Querétaro, Mexico. \\ rdsosam@itesm.mx \\ 2 Krasnow Institute for Advanced Study and Volgenau School of \\ Information Technology and Engineering, George Mason University, USA. \\ john@johngero.com \\ Web page: http://www.computationalcreativity.com/
}

\begin{abstract}
This paper presents a range of computational simulations related to the compatibility of novel ideas that suggest interesting phenomena regarding divergence and convergence, social influence and patterns of change. These computational studies produce insights providing the researcher with another tool to reason about these challenging problems. According to current theory, innovations that are perceived by social groups as having greater compatibility will be adopted more rapidly than other innovations. However, compatibility plays a role in some of the paradoxes of creativity and innovation and its real implications in a range of situations remain unclear.
\end{abstract}

Keywords. Computational social simulation, compatibility, innovative design.

\section{Introduction}

Computational social simulations have been developed to address a number of questions on the link between creativity and innovation [1]. The focus of these studies has been the interaction of the individual level of agency (the change agent) with its social and cultural context in the processes of generation and evaluation of new ideas. Generation and evaluation are regarded as complementary in the dyad novelty-utility found in the canonical definition of creativity. The term "creative situations" captures this assumed coupling or alignment at two levels of agency, namely the individual factors and the contextual conditions, resulting in:

a) a match between individual attributes and actions within the appropriate context in order to generate novel ideas, and

b) the relevant environmental processes that facilitate diffusion, adoption and advantageous consequences of innovations. 
In a) a range of individual and micro-level factors are involved: the preparation and expertise of different individuals, their various abilities to perceive and adequately formulate problems, their access to positions that enable implementation of and experimentation with ideas, initial support for diffusion, etc. This range of factors is directly related to the generative phase of novel ideas. In b) a number of social and macro-level factors are involved: a corpus of predecessors' achievements, information dissemination channels, social perceptions of problems, solutions and drawbacks, norms and practices, production and distribution infrastructure, cultural constraints, etc. This range of factors is directly related to the evaluative phase of novel ideas by a social group.

Whilst factors in a) can be associated with "logic and genius" in Simonton's model of creativity [2], b) provides a level to capture processes of "chance and zeitgeist" in that same model. The concept of creative situations [1] proposes that a type of alignment is necessary between these levels of agency to enable the generation and the evaluation of creative and innovative ideas. Computational social simulations provide useful means to grow these types of theoretical constructs [3].

\section{I.I Compatibility}

According to current theory, innovations that are perceived by social groups as "having greater relative advantage, compatibility, trialability, observability, and less complexity will be adopted more rapidly than other innovations" [4]. Compatibility is an interesting topic of study because it plays a role in some of the paradoxes of creativity and innovation [1]:

- Original ideas may require freedom at many levels, yet constraints - such as compatibility with previous solutions and infrastructure - can actually benefit creativity and innovation $[2,5]$.

- The adoption of new ideas tends to increase as they mature. High quality and commercial success are usually found not in radical innovative ideas, but in more compatible modifications, such as "second generation" products [6].

- High compatibility may cause technological innovations to be more successful (higher adoption and diffusion degrees), yet the opposite may be true for artistic innovations, where the expectation is to break away from current standards [4]. What level of compatibility would better predict the success of a new design idea?

This paper addresses the relationship between compatibility and innovation via computational social simulations, aimed at clarifying or reformulating these apparent contradictions. A model is presented based on the DIFI framework of creativity [7, 8] and the FBS design prototype schema [9]. Our integrative framework is based on the complementarity of generative and evaluative processes by individuals and groups in design $[1,10]$.

Compatibility is conceptually defined here as the degree to which a novel idea shares attributes or properties with dominant or competing ideas. This can be expressed in several ways in any given computational implementation, for instance if 
the designs generated and evaluated by agents are represented numerically, their compatibility can be calculated by their shared numerical attributes. If the designs are represented by geometrical shapes, compatibility can be given by their shared geometrical attributes, possibly as they are perceived by a group of agents.

\section{Framework}

This section describes the conceptual architecture of our framework rather than the technical implementation details which can be found elsewhere [1]. This enables us to place a stronger emphasis here on the types of hypotheses embedded in the framework, the types of experiments carried on these studies, and the types of results obtained.

Computational social simulation refers to the study of social agency through the ideation, implementation, and execution of computer models usually built under rather simple assumptions with which the experimenter is able to define a series of hypotheses and formally implement and experiment with them to explore the consequences of their interaction over time.

The type of computational systems that we have built in recent years have centred on the idea of social groups (implemented as multi-agent systems or cellular automata) whose members interact in order to generate and evaluate a range of ideas. 'Ideas' can be represented here by numeric values or geometrical shapes, and agent behaviour involves the exchange of values or perceptions of shapes between agents. This enables the modelling of societies where some agents aim to introduce novel ideas that are subsequently valued by their social groups.

In simple models (i.e., cellular automata), an explanatory limit of causality is quickly reached, given that randomness importantly influences the generation of values and their dissemination in constrained spaces of interaction, i.e. typically twodimensional rectangular grids. Interesting variations include experimentation with other types of spaces, but a rather more useful approach involves modelling "bigger" agents in rich social spaces. Typically this means that randomness is replaced by a more grounded approach to guide the processes of generation and evaluation of ideas.

We have thus implemented multi-agent systems where ideas are represented as two-dimensional geometrical shapes that some agents (designers) generate and the rest (societies) perceive, evaluate and ultimately adopt or reject. This is implemented by individual mechanisms of shape perception including geometrical properties like boundaries, number of sides, angles, and transformations like uniform and nonuniform scale, rotation, etc.

The social spaces where agents interact is also enriched by including mechanisms of social influence in various dimensions: societies converge and diverge over time re-shaping groups of agents that share preferences, perceptions and/or decisions regarding existing ideas. Figure 1 shows the system architecture used in this paper as a framework to study compatibility with three main interacting system elements: the individual agent (designer), a social evaluative group (field), and their environment or domain $[1,10]$. 


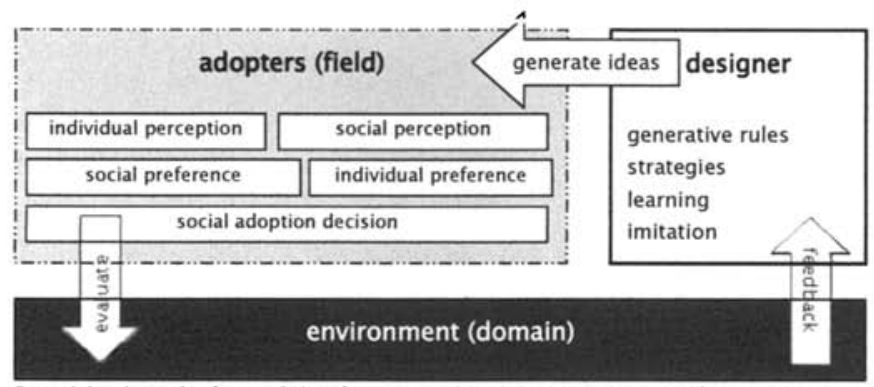

Fig. 1. Graphic description of the framework with three interacting elements: the individual agent (designer), a social evaluative group (field), and environment (domain).

\subsection{Domain-Field-Individual}

In this framework, the domain represents the set of values or ideas shared by a field. It typically includes the competing ideas in a social group at time $t_{i}$ as well as a cumulative number of ideas selected by the society during the simulation, $t_{0<i}$. In our multi-agent models the domain is usually implemented as a dynamic array where successful ideas are stored, possibly with a rate of decay representing the lifespan of the 'collective memory' of a society. Inclusion of ideas into the domain is implemented by a bottom-up mechanism by which agents that influence others gradually gain authority until a few of them exert the role of 'gatekeepers' of the domain. We have explored a range of possible mechanisms observing different emerging patterns of gatekeeping [10].

The field is defined in this framework by the aggregate characteristics of the agents and their interaction over time in different social spaces. In each of these spaces, cycles of convergence and divergence can be 'grown' as in Axelrod's classical model of influence [11]. The adoption decisions can be constrained by the confluence of these social spaces. The implementation of a social space can consist of running a cycle regulating all agent interactions by a given criterion. In a social space of preferences, agents may exchange or influence each other's bias towards certain geometrical features in their adoption decisions; in a space of perceptions, agents may exchange or influence each other's attributes of competing shapes. Experimental settings here include modifying the rate of exchange at different spaces, the interaction rules, and the type of data structure used in the implementation.

Lastly, the individual agent is defined in this framework by the set of design rules carried by the agents that besides evaluation are able to generate new or modify existing shapes, which are subsequently available for social evaluation. Here the range of experimental settings is large and includes generative mechanisms, competition strategies, novelty seeking motivation, distributions of traits and abilities, rates of creation, etc. The role of the individual agent can be implemented via an evolutionary system, analogy making, case-based reasoning or any other generative process potentially including direct human participation, although we have not yet explored this hybrid approach. An implementation of this framework can make use of a geometrical shape representation that captures some of the 
properties of design solutions. Moreover, this representation supports reasoning mechanisms for adoption decisions based on the geometrical properties of sets of two-dimensional line representations constrained by 12 boundary points as shown in Figure 2(a). This is a simple way of representing features of design ideas with nomological constraints.

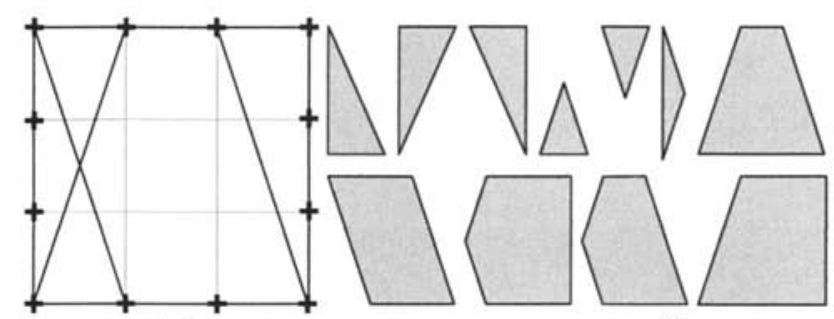

(a)

(b)

Fig. 2. (a) A simple geometrical shape perception and (b) some possible interpretations built by different adopters based on individual perception biases.

Multiple representation and ambiguity are possible because ideas are perceived and interpreted by adopters according to a set of randomly distributed perception biases. Figure 2(b) shows sample perceived features of an idea. The assumption is that people perceive design ideas in (marginally) different ways and therefore base their evaluations on different features of those ideas. By manipulating experimental variables at the domain, field and individual levels, we are able to explore in our computational models the formation of patterns over simulated time of social influence, diffusion, and emergence of new values. We have commenced by manipulating a few variables independently, registering their effects and assessing the framework's ability to capture phenomena observed in field and laboratory studies published in the literature [1].

\subsection{A Simulation Run}

The role of designers is modelled here as instances of change agents that work towards providing novel solutions to a set of problems shared by large social groups. Typically, in these social simulations a small set of up to half a dozen designers compete by iteratively interpreting the problem and proposing a solution which is evaluated by the whole social group including other designers. The designer agents learn from the feedback provided by the social group including their adoption decisions and a measure of satisfaction with their adopted solutions. Designer agents also have a learning mechanism that influences their future behaviour based on the overt actions of their competitors and the social adoption of their solutions. Although the evaluation process carried by adopter agents follows a set of rules that define individual perception and preferences (following a normal distribution), social interaction is included as the potential of adopter agents to influence each other's decisions to adopt or reject solutions generated by the designers. 
Three social spaces are implemented in the studies reported in this paper: a space where geometrical preferences are exchanged, a second space where shape perceptions are exchanged, and a third space where adoptions decisions are exchanged. In social groups of a few hundred adopters, patterns of interest arise such as the emergence of opinion leaders and cycles of convergent-divergent adoption. During a simulation, the system is set to track the behaviour of every agent as well as the global patterns of group behaviour. Despite their apparent simplicity, these models of co-evolution generate non-linear effects that emerge from the interaction of their components over time. In this way, researchers are equipped with in silico laboratories where they can 'grow up' different states from a set of initial conditions, gaining insights into the role of designers as change agents in complex systems.

\subsection{Compatibility Studies}

In simple cellular automata models of social influence, compatibility has been identified as a key determinant of interaction [11]. Global group convergence tends to emerge as the aggregate effect of distributed local exchanges based on the gradual development of regions of compatible values. Starting from random conditions, the group tends to converge in one dominant value or reach a lock-in state where regions of incompatible values emerge. These systems have been extensively replicated showing that the final outcome of group convergence is highly likely depending on key variables such as the range of values assigned and the rules of interaction between neighbours or adjacent cells. These variations determine the likelihood of compatibility between cells in the grid and between regions of cells. If adjacent regions in a cellular automata develop compatible values, it is inevitable that a single dominant value will emerge either by dominance of one region over the others, or by combination of their compatible values. If incompatibility occurs, interaction is halted across regions and global convergence is not reached.

In a multi-agent system implementation of the framework presented in this paper where domain, field and individual design agents interact, it is possible to inspect the concept of compatibility further. Using an idea representation like the one described earlier based on geometrical shapes, compatibility can be measured as a degree of similarity. Figures 3(a) to 3(d) show a range of shape perceptions with different indices of compatibility based on shared geometrical characteristics. Figure 3(a) and 3(b) are more compatible since they share seven line segments, whilst Figure 3(a) and $3(\mathrm{c})$ are less compatible since they have only three line segments in common. In the same vein, Figures 3(b), 3(c) and 3(d) are compatible because they share symmetric properties, whilst Figure $3($ a) is incompatible symmetry-wise. Likewise, Figures 3(a) and 3(b) are compatible in that they both present right angles, whilst Figures 3(c) and 3(d) do not. 


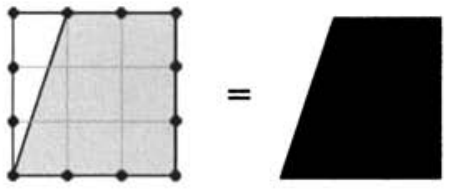

a)

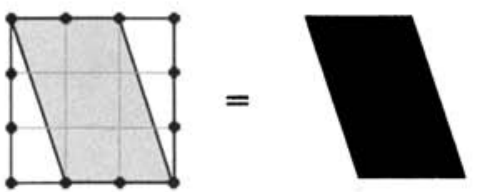

c)

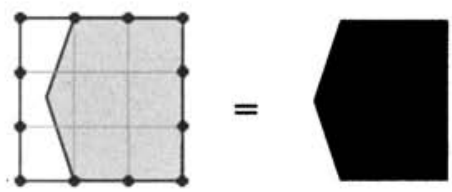

b)

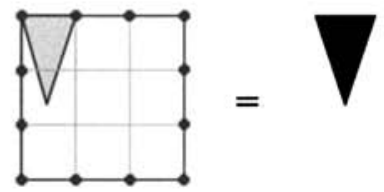

d)

Fig. 3. Compatibility between ideas is implemented here based on the shared geometrical properties of shapes. Number of line segments, angles, symmetry, and other properties can be incorporated by evaluating agents to determine the degree of compatibility in any set of shapes.

Compatibility can evolve during a simulation run for any agent in relation to any given shape due to agent interaction in different social spaces. This is possible due to the constantly evolving preferences, perceptions and adoption decisions being continuously exchanged in the social group and periodically updated due to the introduction of new ideas by the design agents.

The following are the experimental settings explored in this paper:

1. Conditions are first explored in relation to compatibility and adoption of new ideas. Monte Carlo simulations traverse the idea compatibility space. This is implemented by running simulations with identical initial conditions in all control variables except the generative processes of designers, which are manipulated to generate new ideas that go from entirely incompatible to entirely compatible, namely $0<c<1$, where $c$ is the degree of compatibility as estimated by the designer agent introducing the idea into the system. This corresponds to one extremum where new ideas are entirely random to the other where new ideas are identical to existing ideas (at least from the designer's viewpoint, which can be marginally different as measured by some social groups which operate with varying perceptions). The results of the system at the three levels are recorded on every simulation run for every initial condition (results represent an average over ten runs for every step in the parameter space). The field effects of compatibility is analysed, i.e., the adoption patterns.

2. A slight variation addresses the effect that compatibility has on the type of innovation observed in a society. The assumption is that, with all other conditions kept constant, changes in compatibility of new ideas may yield an output of either radical or transformational innovations in the system. In line with the literature, radical innovations are characterised by the qualitative differences between succeeding dominant ideas in a society. Low levels of compatibility may yield radical innovations, whilst marginal differences may 
emerge from generative processes that promote high compatibility. This is implemented by Monte Carlo simulations traversing the space of compatibility in the generative process. The focus in this case is in the analysis of the resulting domain.

3. A second aspect of interest is competition. Previous studies have suggested that the rate of the generative processes of new ideas may have a non-linear effect on innovation [1]. This is explained by a "glass ceiling" that imposes a limit on the frequency and scope of cycles of change, due to the time required for new ideas to be disseminated. It is not clear what could be the effects of compatibility and rate of generation of new ideas. This is implemented by Monte Carlo simulations traversing the spaces of compatibility and rate of behaviour by designer agents.

4. A third question is addressed regarding compatibility and complexity. Theories suggest that innovations are more effective if new ideas are more compatible and less complex [4]. Our framework enables experimentation by traversing the compatibility and complexity spaces of new ideas in the generative processes. As in the previous settings, all other conditions are kept constant, whilst the generative processes of designer agents in the system are controlled at the initial time enabling analysis on the outputs at domain and field levels when a) compatibility is low and complexity is high, b) compatibility is high and complexity is low, c) both are high, and d) both are low. Complexity in this framework can be measured by the length of the representation of the geometrical shapes. One key assumption here is that more complex shapes will enable a higher diversity of perceptions of new ideas by members of the social group.

\subsection{Results}

A set of key implications result from our simulations related to the compatibility of novel ideas introduced in a social group.

1. Low levels of compatibility may yield high levels of divergence in a social group, causing information flow to stop and thus, precluding innovation. This has been characterised in equivalent modelling approaches as the emergence of "few or many distinct cultural regions depending on the scope of cultural possibilities, the range of interactions, and the size of the geographic territory" [11].

2. High levels of compatibility may cause total and rapid convergence in a social group. Whilst constant cycles of change take place under such conditions, the impact of novel yet highly compatible ideas is minimal. Namely, such simulations typically show continuous cycles of 'transformational' innovations, i.e., where small variations of a dominant idea are repeatedly introduced.

3. If novel ideas with low levels of compatibility are introduced in a social group, but information flow is sustained during long time periods (externally or otherwise), a high rate of crossover of ideas is likely. In such cases, periodical cycles of change have large impacts (changes are significant and have large scope). In addition, such cases show that the 'culture' of a society may change radically even if social structure remains unchanged. 
4. Low levels of compatibility may yield opportunistic innovations if the rate of idea production is high enough to support a competitive environment. An opportunistic innovation is defined here as the wide adoption of a new idea that draws attributes from competing new ideas, maintaining their advantage but increasing their compatibility.

5. A general consensus in the literature is that high compatibility combined with low complexity yield relatively fast diffusion rates and a reasonable scope of diffusion [4]. In our framework, less complex designs are those that can be represented with a smaller range of attributes, numerical, geometrical or otherwise. Our simulations illustrate that compatibility and complexity may exhibit undesirable effects given that in solutions with very low levels of complexity, a small attribute variation between two designs can rapidly decrease their compatibility. In contrast, high levels of complexity support a large variance of solutions marginally differentiated and thus, solutions with high levels of compatibility. Therefore, a balance between high compatibility and low complexity may be hard to achieve, accounting for their exceptional joint occurrence.

\section{Discussion}

A key potential implication of these studies is that isolated characteristics of designers and their ideas are insufficient to formulate conclusions about creativity and innovation. Causality may rather be inspected in the situational factors that define the relationship between designers and their evaluators. This framework enables the study of compatibility and innovation from a situational viewpoint, suggesting ways in which key characteristics of innovations may have very different causes and consequences depending on the surrounding contextual conditions. The following design guidelines can be formulated:

1. Design solutions must be perceived as having an adequate degree of compatibility with previous or competing alternatives.

2. In designing innovative solutions, the likely rate of diffusion must be estimated in order to adjust the degree of compatibility to avoid rapid, unstable and difficult to control flows that prevent assimilation of novel ideas.

3. The degree of compatibility of novel ideas may determine the extent to which novel ideas are reinterpreted or combined with existing dominant ideas. In some cases it may be desirable to allow for crossover, whilst in other cases (i.e., intellectual property) this may need to be avoided. This may be addressed by the relation between complexity and compatibility of novel and old ideas.

The computational exploration of compatibility and its interplay with complex phenomena like creativity and innovation yields promising results. Emergence is a key aspect to understand phenomena such as "creative situations". The results are not easily predictable, neither are they definite or necessarily valid against external conditions. Rather, these studies provide insights that provide the researcher with another tool to reason about these challenging problems. One way to advance this 
research methodology would be to contrast these findings with documented cases in the literature, and as aids to design experimental settings in the laboratory or the field.

\section{References}

1. R. Sosa, Computational Explorations of Creativity and Innovation in Design, PhD Thesis, Key Centre of Design Computing and Cognition (University of Sydney: Sydney, 2005).

2. DK. Simonton, Creativity in Science: Chance, Logic, Genius, and Zeitgeist (Cambridge University Press: Cambridge, 2004).

3. JM. Epstein, Generative Social Science: Studies in Agent-Based Computational Modeling (Princeton University Press: New Jersey, 2007).

4. EM. Rogers, Diffusion of Innovations (The Free Press: New York, 1995).

5. D. Partridge, and J. Rowe, Computers and Creativity (Intellect: Oxford, 1994).

6. H. Petroski, The Evolution of Useful Things (Knopf: New York, 1992).

7. M. Csikszentmihalyi, in: The Nature of Creativity, Contemporary Psychological Perspectives, edited by RJ Sternberg (Cambridge University Press, 1988), pp. 325-339.

8. DH. Feldman, M. Csikszentmihalyi, and H. Gardner, Changing the World: A Framework for the Study of Creativity (Praeger: Westport, 1994).

9. JS. Gero, Design Prototypes. A Knowledge Representation Schema for Design, AI Magazine. Volume 11, Number 4, pp. 26-36 (1990).

10. R. Sosa, and JS. Gero, in: Computational and Cognitive Models of Creative Design VI, edited by JS. Gero, and ML. Maher (University of Sydney: Sydney, 2005), pp. 19-44.

11. R. Axelrod, The Dissemination of Culture: A Model with Local Convergence and Global Polarization, The Journal of Conflict Resolution. Volume 41, 2, pp. 203-226 (1997). 